\title{
An experimental and theoretical study of unsteady flow (gust) effects on structures
}

\author{
Ch. Sreenadh, A. Sanz-Andrés, S. Franchini \& F. Navarro-Medina \\ Instituto Universitario de Microgravedad 'Ignacio da Riva' (IDR), \\ Universidad Politécnica de Madrid, Spain
}

\begin{abstract}
The gust wind tunnel at IDR, Universidad Politécnica de Madrid (UPM), has been enhanced and the impact of the modification has been characterized. Several flow quality configurations have been tested. The problems in measuring gusty winds with Pitot tubes have been considered. Experimental results have been obtained and compared with theoretically calculated results (based on potential flow theory). A theoretical correction term has been proposed for unsteady flow measurements obtained with Pitot tubes. The effect of unsteady flow on structures and laying bodies on the ground has been also considered. A theoretical model has been proposed for a semi-circular cylinder and experimental tests have been performed to study the unsteady flow effects, which can help in clarifying the phenomenon.

Keywords: hot wire anemometer, gust wind flow, Pitot tube measurements, gust wind tunnel correction factor for Pitot tubes, unsteady flow measurements, ballast pickup, influence of gusty winds, gust characterization, unsteady flow's effects on structures.
\end{abstract}

\section{Introduction}

For a long time, study of wind gusts and their effects on various bodies lying on the ground has been receiving a strong interest from worldwide researchers. As a part of this interest, IDR/UPM has developed a gust wind tunnel to study and research those effects (fig. 1). The theoretical and conceptual research that justifies the development of the gust wind tunnel can be found in SanzAndres and Navarro-Medina [1]. 


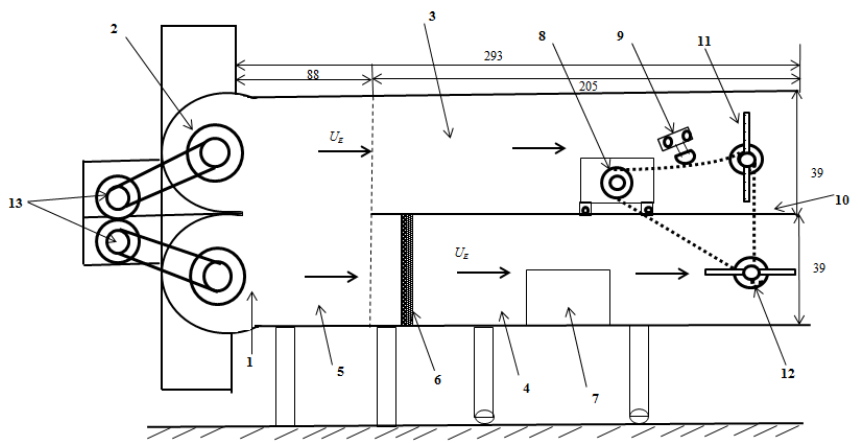

Figure 1: $\quad$ Schematic diagram of the gust wind tunnel with the gust generation mechanism (dimensions in $\mathrm{cm}$ ). (1) Centrifugal fan-1, (2) Centrifugal fan-2, (3) Test chamber-1, (4) Test chamber-2, (5) Transition chamber , (6) Wind flow quality filters, (7) Testing area, (8) DC motor, (9) Chain tensor, (10) Test chamber splitter board, (11) Rotating gate-1, (12) Rotating gate-2, (13) Fans driving motors. (Note: gate rotation frequency, $f_{g}{ }^{N}$, is measured by an optical laser sensor (not shown in this fig. 1).)

The description of the gust wind tunnel can be found in Navarro-Medina et al. [2]. In the initial version of this gust wind tunnel, the gust frequency control had not an electronic feedback. In the present wind tunnel an electronic control system (INFRNOR, model SQC-2I) for the gust generation DC motor (MAVILOR-MO-1000 STD, model $1120 \mathrm{~W}$ ) is included.

In unsteady, accelerating, or fluctuating fluid flows, due to the inertial effects of the fluid, Pitot tubes cannot accurately measure the fluid flow (Pratt [3]). To overcome this problem a theoretical correction is proposed here to correct the measurements of the Pitot tube.

The problems in unsteady or gusty flow on structures have been considered by Naudascher and Rockwell [4] by using a semi-circular cylinder. A similar body is considered in the present paper. According to [4], in unsteady flow, a flow passing around a semi-circular cylinder the phenomenon of wake breathing takes place. A semi-empirical theoretical model is proposed here, based in a twodimensional potential flow model, correcting the wake effect, which is validated with the experimental results.

This paper is organized as follows. In section 2, an experimental setup to characterize the unsteady uniform flow with a hot-wire anemometer and a Pitot tube and an experimental setup to steady the flow around a semi-circular cylinder are presented. In section 3, a brief description of the testing procedure is described. In section 4, the characterization of the gust in the wind tunnel, is described. In section 5, the proposed theoretical correction term, Pitot tube's measurements and simulated pressure (based on the hot-wire anemometer measurements) are presented. In section 6, a theoretical model of the unsteady flow around the semi-circular cylinder together with experimental results is 
described. In section 7, results are summarized. Finally, in section 8, conclusions are drawn.

\section{Experimental setup}

In this section a brief description of, the hot-wire anemometer probe and Pitot tube and hot-wire anemometer probe and semi-circular cylinder experimental setups are presented.

\subsection{Hot wire anemometer probe and Pitot-tube}

For measuring the gust wind flow inside the test chamber, a hot-wire anemometer with probe, (a mini CTA version 4.10 of Dantec Dynamics [5]) and a Pitot tube with a pressure transducer $\mathrm{TR}_{1}$, (Sensor Technics, model BTEL 5905 DIA,) are mounted in the test section. The hot-wire anemometer probe (fixed to a wooden supporting stand) and Pitot tube have been placed at $19.5 \mathrm{~cm}$ above the base of the test section-2 (fig. 2(a)).

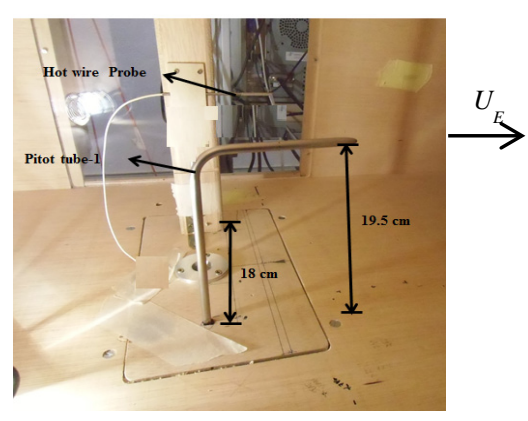

(a)

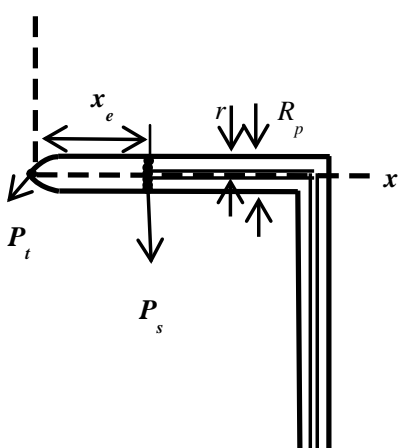

(b)

Figure 2: (a) Experimental setup of the Pitot tube and hot-wire probe in test section; (b) Schematic diagram of the Pitot tube with geometrical nomenclature.

The output from the hot-wire anemometer probe is connected to the input of the mini-CTA. The outputs from the mini-CTA and Pitot tube's pressure transducer, $\mathrm{TR}_{1}$, are connected to a desktop computer through a connection hub (Model BNC: 2110, National Instruments). The application software Streamware (Dantec Dynamics [5]), installed in the desktop computer, transforms the readings from the hot-wire anemometer to measured fluid speed, $U_{E}{ }^{M}$, in the test section. For the case of Pitot tubes, pressure transducer, $\mathrm{TR}_{1}$, generates the pressure difference $\Delta p$. From the measured $\Delta p$ the speed of the measured fluid flow, $U_{E}{ }^{M}$, is estimated by using the Bernoulli's equation (in the case of steady flow) 


$$
U_{E}=\sqrt{\frac{2\left(p_{t}-p_{s}\right)}{\rho_{a}}} .
$$

The air density, $\rho_{a}$, obtained from the ambient conditions.

\subsection{Semi-circular cylinder}

To study the unsteady flow effects on structures lying on the ground, a semicircular cylinder (radius $0.05 \mathrm{~m}$ ) and a hot-wire anemometer probe are mounted in the test section (fig. 3 ). The semi-circular cylinder includes 3 pressure taps, $P_{0}$, $P_{1}$ and $P_{4}$. The pressure differences $\mathrm{P}_{1}-\mathrm{P}_{0}$ and $\mathrm{P}_{4}-\mathrm{P}_{0}$ are measured by the pressure transducers $\mathrm{TR}_{1}$ and $\mathrm{TR}_{2}$ (Honey well, model 1639CO1D75) respectively. The mean value of the flow speed measured by hot-wire anemometer is used as a reference flow speed, $U_{E}$, in the test section.

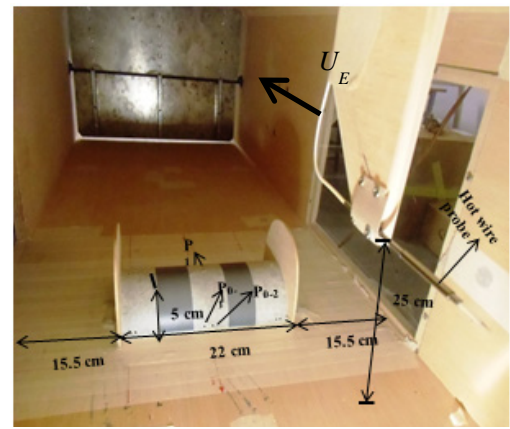

(a)

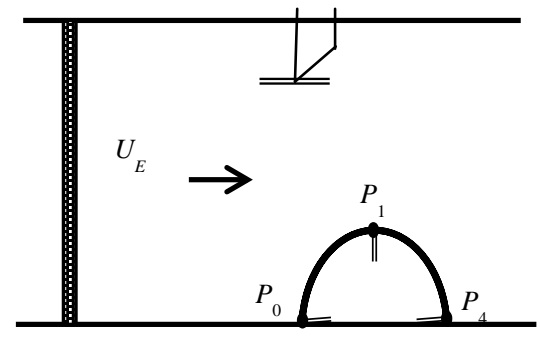

(b)

Figure 3: (a) Hot-wire anemometer probe and semi-circular cylinder experimental setup; (b) Schematic diagram of the semi-circular cylinder experimental setup.

\section{Testing procedure}

For improving the wind flow quality inside the test section, one of the configurations listed in table 1 can be selected (Navarro-Medina [2]).

In this paper, the configuration $\mathrm{C}_{4}$ has been selected as the best wind flow quality in the test section. The other parameters nominal gust frequency, $f_{g}{ }^{N}$, = $\left[0,2,4,6\right.$, and $8 \mathrm{~Hz}$ ], fan speed, $f_{m}=30$ and $40 \mathrm{~Hz}(60 \%$ and $80 \%$ of the power supply of fan motors) and $T_{g c}=380 \mathrm{~mm}$ (rotating gate chord maximum blockage of a test section area) have been chosen for these experiments. The test time duration has been set to 2 minutes for each value of nominal gust frequency, $f_{g}{ }^{N}$. (In the following sections, to have a better view of the results, all the plots are plotted for first one second of the recorded data). For checking the measuring instruments, in the steady flow, a sample test data was recorded. The measurement data obtained from the hot-wire anemometer and Pitot tube, PT, 
have been processed by a Matlab program. Results are shown in fig. 4 and discussed in section 7 .

Table 1: Wind flow’s quality configurations.

\begin{tabular}{|c|l|}
\hline Symbol & \multicolumn{1}{|c|}{ Description } \\
\hline $\mathrm{C}_{1}$ & $\begin{array}{l}\text { A clear entrance for flow towards test section-2 (no flow } \\
\text { quality enhancement). }\end{array}$ \\
\hline $\mathrm{C}_{2}$ & $\begin{array}{l}\text { A } 50 \mathrm{~mm} \text { wide honeycomb screen with } 5 \mathrm{~mm} \text { cells, placed at the entrance } \\
\text { of flow towards test section- } 2 .\end{array}$ \\
\hline $\mathrm{C}_{3}$ & $\begin{array}{l}\text { Honeycomb of previous configurations plus a } 5.2 \mathrm{~mm} \text { cell square grid of } \\
0.8 \text { mm porosity, made of } 0.7 \mathrm{~mm} \text { diameter wire, placed just downstream. }\end{array}$ \\
\hline $\mathrm{C}_{4}$ & $\begin{array}{l}\text { Configuration } \mathrm{C}_{2} \text {, adding a } 15 \mathrm{~mm} \text { wide foam screen placed upstream } \\
\text { of the honeycomb. }\end{array}$ \\
\hline
\end{tabular}

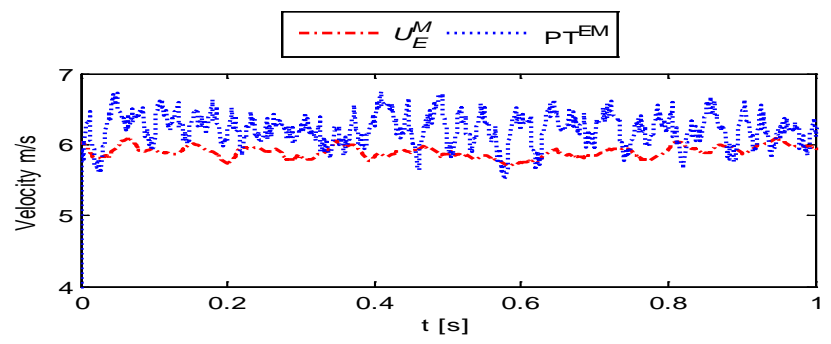

Figure 4: Plot of the velocity meaured with the hot-wire, $U_{E}{ }^{M}$, Pitot tube, $\mathrm{PT}^{\mathrm{EM}}$ as a function of time [s] in steady flow with configuration $\mathrm{C}_{4}$, rotating gate chord, $T_{g c}=380 \mathrm{~mm}$, nominal gust frequency, $f_{g}{ }^{N}=0$ $\mathrm{Hz}$ and fan speed, $f_{m}=30 \mathrm{~Hz}$.

\section{Characterization of the gust wind}

This section describes the characterization of the experimental gust data. The gusty wind speed, $U_{E}{ }^{M}$, is fitted to a sinusoidal function, $U_{E}{ }^{F}$

$$
U_{E}^{F}=A \sin (\omega t+\varphi) .
$$

where $A$ is the amplitude, $\omega=$ the angular frequency and $\varphi$ the phase .

It has been observed that the experimental data of the hot-wire measurements of the fluid flow, namely the velocity, closely follows a sinusoidal waveform. By the application of the Matlab program's data curve fitting option, the measured hot-wire's velocity data, $U_{E}{ }^{M}$, have been fitted to the sinusoidal form, $U_{E}{ }^{F}$. The experimental and fitted data of the test with configuration $\mathrm{C}_{4}$, rotating gate chord, $T_{g c}=380 \mathrm{~mm}$, nominal gust frequency, $f_{g}{ }^{N}=8 \mathrm{~Hz}$ and fan speed, $f_{m}=30 \mathrm{~Hz}$, are 
plotted in fig. 5. The fitted experimental data parameters $\left(A, \omega, \varphi, f_{g}{ }^{F}=\omega / 2 \pi\right)$ ), are listed in the table 2, together with $f_{g}^{N}$ (measured by an optical laser sensor) and regression parameter, $\mathrm{R}^{2}$. The results are discussed in section 7 .

Table 2: $\quad$ Results of fitting the hot-wire measured data, $U_{E}{ }^{M}$, to a sinusoidal function, $U_{E}^{F}$, according to eqn. (2) with fan speed, $f_{m}=30 \mathrm{~Hz}$.

\begin{tabular}{|c|c|c|c|c|c|}
\hline $\begin{array}{c}A \\
{[\mathrm{~m} / \mathrm{s}]}\end{array}$ & $\begin{array}{c}\varphi \\
{[\mathrm{m} / \mathrm{s}]}\end{array}$ & $\begin{array}{c}\omega \\
{[\mathrm{rad} / \mathrm{s}]}\end{array}$ & $\begin{array}{c}f_{g}{ }^{N} \\
{[\mathrm{~Hz}]}\end{array}$ & $\begin{array}{c}f_{g}^{F} \\
{[\mathrm{~Hz}]}\end{array}$ & $\mathrm{R}^{2}$ \\
\hline 2.07 & -0.13 & 14.74 & 2.00 & 2.35 & 0.97 \\
\hline 2.02 & 3.49 & 25.71 & 4.00 & 4.09 & 0.98 \\
\hline 1.89 & -0.02 & 38.91 & 6.00 & 6.20 & 0.98 \\
\hline 1.63 & 1.28 & 48.20 & 8.00 & 7.70 & 0.99 \\
\hline
\end{tabular}

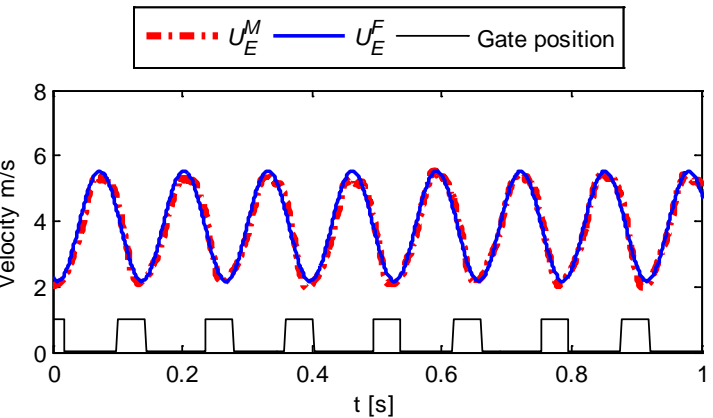

Figure 5: Plot of the hot-wire measured velocity, $U_{E}{ }^{M}$, curve fitted function eqn. (2), $U_{E}{ }^{F}$, and rotating gate position (1: fully open, 0:closed) as a function of time [s] in unsteady flow with configuration $\mathrm{C}_{4}$, rotating gate chord, $T_{g c}=380 \mathrm{~mm}$, nominal gust frequency, $f_{g}{ }^{N}=8$ $\mathrm{Hz}$ and fan speed, $f_{m}=30 \mathrm{~Hz}$.

\section{Theoretical correction factor for Pitot tube measurements in unsteady fluid flow}

In this section, a theoretical correction term, $T_{c t}$, to the Pitot tube measurements in unsteady flow (Pratt [3]) is presented. A general schematic diagram of the Pitot tube with geometrical parameters is shown in fig. 2b. From the potential flow theory (Meseguer and Sanz-Andres [6], Batchelor [7]) the pressure correction term, $T_{c t}$ and the simulated Pitot tube pressure, $P_{\mathrm{HWA}}$, (from the hotwire velocity pressure, $q_{H W A}$, with correction term $T_{c t}$ ) can be defined as

$$
T_{c t}=\rho_{a} \dot{U_{E}^{F}} x_{e \mathrm{PT}}, \quad P_{\mathrm{HWA}}=\rho_{a} \dot{U_{E}^{F}} x_{e \mathrm{PT}}+\frac{1}{2} \rho_{a}\left(U_{E}^{F}(t)\right)^{2},
$$




$$
q_{H W A}=\frac{1}{2} \rho_{a}\left(U_{E}^{F}(t)\right)^{2}, \quad U_{E}^{F}=A \sin (\omega t+\varphi), \dot{U_{E}^{F}}=A \omega \cos (\omega t+\varphi),
$$

where $U_{E}^{F}$ is the fitted data of the unsteady fluid flow velocity, $U_{E}{ }^{M}, \rho_{a}$ is the air density, $T_{c t}$ the correction term, $x_{e \mathrm{PT}}$ the geometrical distance between total and static pressure measuring points (shown in fig. 2(b)). $\mathrm{PT}^{\mathrm{EM}}$ is the Pitot tube measured pressure difference. The plot of, $q_{H W A}, P_{H W A}, \mathrm{PT}^{\mathrm{EM}}$ are plotted in fig. 6 . Results are discussed in section 7.

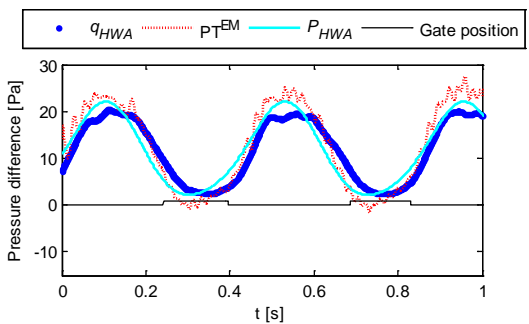

(a): $f_{g}^{N}=2 \mathrm{~Hz}$.

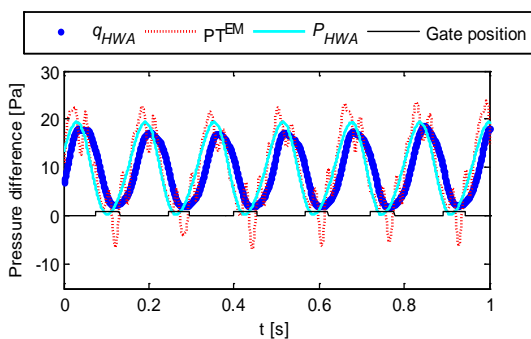

(c) $f_{g}^{N}=6 \mathrm{~Hz}$.

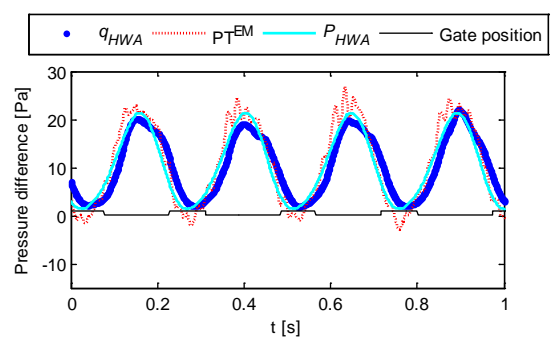

(b): $f_{g}^{N}=4 \mathrm{~Hz}$.

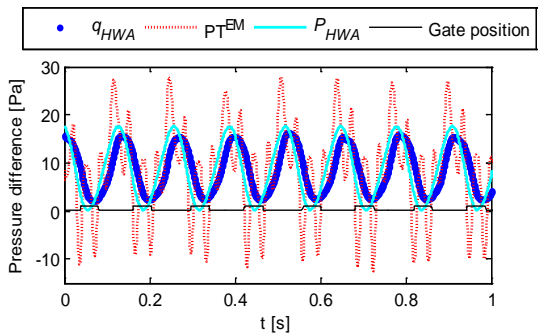

(d) $f_{g}{ }^{N}=8 \mathrm{~Hz}$.

Figure 6: $\quad$ Plot of the $q_{H W A}, \mathrm{PT}^{\mathrm{EM}}$ and $P_{\mathrm{HWA}}$ and gust generating gate position (1: is fully open, 0 : closed) $\left(x_{e \mathrm{PT}}=0.06 \mathrm{~m}\right)$ varies with time function $\mathrm{t}\left[\mathrm{s}\right.$ ], in configuration $\mathrm{C}_{4}$, with rotating gate chord, $T_{g c}=380 \mathrm{~mm}$, nominal gust frequency, $f_{g}{ }^{N}=[2,4,6,8 \mathrm{~Hz}]$ and fan speed, $f_{m}=$ $30 \mathrm{~Hz}$ (i.e. mean flow speed is 5 to $6 \mathrm{~m} / \mathrm{s}$ ).

\subsection{The influence of the static pressure geometrical distance}

From the results of the section 6 , it is observed that the proposed correction term, $T_{c}$, is suitably correcting the Pitot tube measured pressure up to the nominal gust frequency is $f_{g}^{N}=4 \mathrm{~Hz}$ (figs. 6(a) and 6(b)). But it fails if the nominal gust frequency is $f_{g}^{N}>4 \mathrm{~Hz}$ (fig. 6(c) and 6(d)).

To study this problem and check the suitability of the proposed correction term, $T_{c t}$, a new experimental setup (fig. 7) was proposed. In a uniform, unsteady flow, a static pressure tap was placed at the wind tunnel test section- 2 base wall, at different horizontal distances from the total pressure measuring tube nose. The static pressure measuring point positions were, $x_{e \mathrm{PT}}=-10,-5,0$, and $5 \mathrm{~cm}$. Experimental data were recorded and the suitability of the correction term, $T_{c t \text {, }}$ were analyzed. Results are plotted in fig. 8. Results are discussed in section 7. 


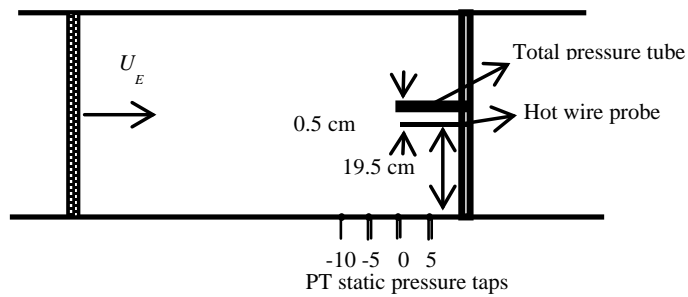

Figure 7: Schematic diagram experimental setup of the Pitot tube and hotwire probe in test section.

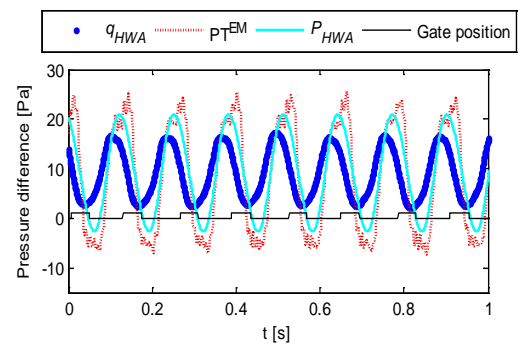

(a): $X_{e \mathrm{PT}}=-10 \mathrm{~cm}$

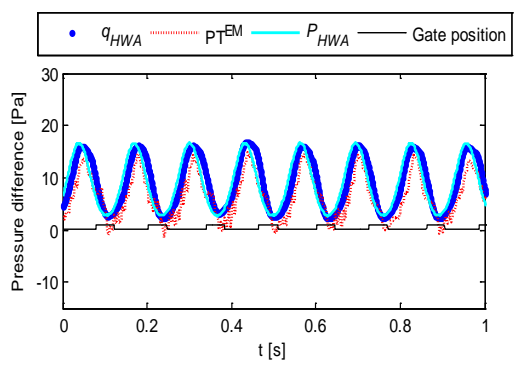

(c): $X_{e \mathrm{PT}}=0 \mathrm{~cm}$

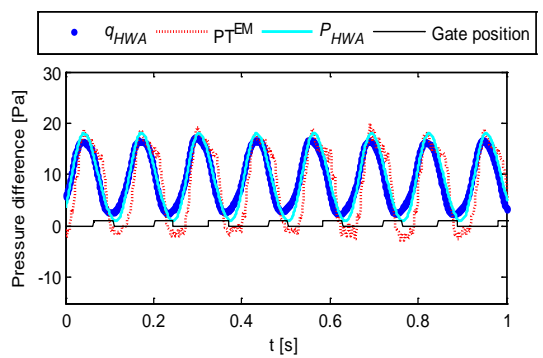

(b): $X_{e \mathrm{PT}}=-5 \mathrm{~cm}$

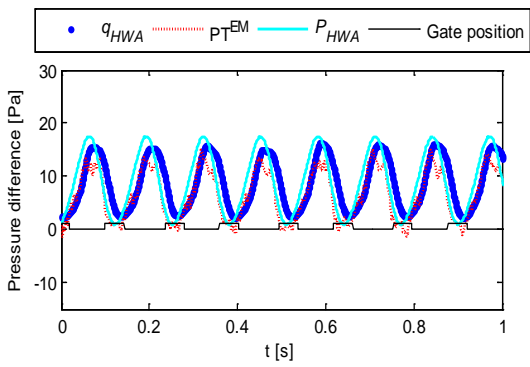

(d): $X_{e \mathrm{PT}}=5 \mathrm{~cm}$

Figure 8: Plot of the $q_{H W A}, \mathrm{PT}^{\mathrm{EM}}$ and $P_{\mathrm{HWA}}$ and gust generating gate position (1: is fully open, 0 : closed) $\left(x_{e \mathrm{PT}}=-10,-5,0,5 \mathrm{~cm}\right)$ varies with time function $\mathrm{t}\left[\mathrm{s}\right.$ ], in configuration $\mathrm{C}_{4}$, with rotating gate chord, $T_{g c}$ $=380 \mathrm{~mm}$, nominal gust frequency, $f_{g}{ }^{N}=8 \mathrm{~Hz}$ and fan speed, $f_{m}=$ $30 \mathrm{~Hz}$ (i.e. mean flow speed is 5 to $6 \mathrm{~m} / \mathrm{s}$ ).

\section{Unsteady flow effects on the semi-circular cylinder}

The results of the measurements performed about the unsteady flow around a semi-circular cylinder are presented in this section, together with a simple mathematical model that can help to analyse the results and to explain some of their characteristics. The model includes both steady and non-steady terms, with the objective of evaluating the relevance of the unsteady effects with regard to 
the quasi-steady loads. The experimental setup is shown in the fig. 3. In the experimental setup, the semi-circular cross section cylinder is placed perpendicular to the mean flow direction. The fluid flow can be considered as 2D close to the mean section of the cylinder, where the pressure taps are placed.

\subsection{Analysis of the steady flow case}

The effects of the steady flow on the pressure field of the semi-circular cylinder are presented in this section. Let us consider the steady flow as a $2 \mathrm{D}$ potential flow around the circular cylinder. The static-pressure referenced coefficient at tap $\mathrm{P}_{1}$ (referenced to the mean flow speed in the test section) is defined as (Clancy [9]),

$$
c_{p e 1}=\frac{p_{1}-p_{e}}{q}, \quad q=\frac{1}{2} \rho_{a} U_{m v}^{2},
$$

where $p_{1}$ is the pressure at tap $1, p_{e}$ is the static pressure of the flow, $q$ is the velocity pressure, $U_{m v}$ the mean value of the flow velocity, $U_{E}$, and $\rho_{a}$ the air density. The values of the pressure coefficients can be, $c_{p e 1}=-1.5,-2,-3$ as far as the flow can be assumed as laminar, turbulent or just completely potential (meaning without detachment in the leeward side (Meseguer and Sanz-Andres [6], Roshko [8])). The static-pressure referenced coefficient at tap $\mathrm{P}_{4}$ (assuming that at P4, it is the base pressure, constant in the near wake of the body) is assumed as,

$$
c_{p e 4}=\frac{p_{4}-p_{e}}{q} \cong-0.5,
$$

from the well-known experimental data (Roshko [8]).

\subsection{Pressure coefficients referenced to the total pressure}

As in an unsteady flow it would be very difficult to measure the static pressure (it is not constant along the flow and change with time). The data obtained from the tests are the pressure differences between the pressure taps $\mathrm{P}_{1}, \mathrm{P}_{0}$ and $\mathrm{P}_{4}, \mathrm{P}_{0}$ (i.e. referenced to the pressure of tap $\mathrm{P}_{0}$ which measures the stagnation pressure). For convenience, let $p_{0}$ be the stagnation pressure of the incoming flow speed $U_{E}$. The data obtained from the experiments, the stagnation-pressure referenced coefficients at the pressure taps $\mathrm{P}_{1}$ and $\mathrm{P}_{4}$ are denoted as, $c_{p 01}, c_{p 04}$ and defined as,

$$
c_{p 04}=\frac{p_{4}-p_{0}}{q}, c_{p 01}=\frac{p_{1}-p_{0}}{q}, \quad q=\frac{1}{2} \rho_{a} U_{m v}^{2} .
$$

\subsection{Analysis of the unsteady flow case}

The two dimensional potential flow of an incoming uniform stream, $U_{E}$, around a circular cylinder can be described by using the complex potential formulation in the classical form (Meseguer and Sanz-Andres [6], Batchelor [7]),

$$
f(\tau)=U_{E}\left(\tau+\frac{R^{2}}{\tau}\right),
$$


where $\tau=x+\mathrm{iz}$ is the complex variable and $R$ is the radius of the cylinder. The velocity on the cylinder surface $\left(\tau=R e^{i \theta}\right)$ is given by

$$
V=\sqrt{1-c_{p e m}} U_{E} \sin \theta=k_{m} U_{E} \sin \theta
$$

where $k_{m}=\sqrt{1-c_{p e 1}}=\sqrt{3}$, or $2 . k_{m} U_{E}$ is the maximum velocity which is measured at pressure tap $\mathrm{P}_{1}$. The pressure is given by the Euler-Bernoulli equation for unsteady potential flow (Meseguer and Sanz-Andres [6], Batchelor [7]),

$$
\frac{p}{\rho_{a}}+\frac{1}{2} U_{E}^{2}+\varphi_{t}=C(t)
$$

where $C(t)$ is uniform all along the fluid field, $\varphi$ can be obtained from eqn. (8)

$$
\begin{aligned}
& \varphi=\operatorname{Re}[f(t)]=U_{E} \operatorname{Re}\left[\left(\operatorname{Re}^{\mathrm{i} \theta}+\operatorname{Re}^{-\mathrm{i} \theta}\right)\right] \\
& =2 R U_{E} \cos \theta=2 U_{E} X,
\end{aligned}
$$

where $\operatorname{Re}[]$ means the real part of the argument. From eqns. (10) and (11) the pressure field acting on the body is given by

$$
\frac{p}{\rho_{a}}=C(t)-\frac{k_{m}^{2}}{2} U_{E}^{2} \sin ^{2} \theta-2 \dot{U}_{E} x,
$$

where the $\dot{U}_{E}$ represents the derivative with time. At the stagnation point ( $\theta=\pi$, $x=-R$ ) the pressure $p_{0}$ is

$$
\frac{p_{0}}{\rho_{a}}=C(t)+2 \dot{U}_{E} R
$$

From eqns. (12) and (13) the pressure referenced to the stagnation pressure is given by

$$
\frac{\left(p_{0}-p\right)}{\rho_{a}}=\frac{k_{m}^{2}}{2} U_{E}^{2} \sin ^{2} \theta+2 \dot{U}_{E}(R+x) .
$$

Experimentally measured stagnation-pressure referenced coefficients, $c_{p 01}^{E}$ and $c^{E}{ }_{p 04}$ are calculated from the measurements $\left(p_{1}-p_{0}\right)$ and $\left(p_{4}-p_{0}\right)$ respective-ly as follows,

$$
c_{p 01}^{E}=\frac{p_{1}-p_{0}}{0.5 \rho_{a} U_{m v}^{2}}, \quad c_{p 04}^{E}=\frac{p_{4}-p_{0}}{0.5 \rho_{a} U_{m v}^{2}}, \quad c_{q_{\mathrm{HWA}}}^{E}=\frac{0.5 \rho_{a} U_{E}^{2}}{0.5 \rho_{a} U_{m v}^{2}}=\frac{U_{E}^{2}}{U_{m v}^{2}} .
$$

$c^{E}{ }_{q \mathrm{HWA}}$ is the velocity pressure obtained from hot-wire measurements and dimension less with the reference velocity pressure. The theoretical predictions for the stagnation -pressure coefficients, taking in to account an unsteady incoming speed $U_{E}(t)$, from the eqns. (7) and (14)

$$
c_{p 01}^{T}=-\frac{U_{E}^{2}}{U_{m v}^{2}}\left(k_{m}^{2}+4 \frac{\dot{U}_{E} R}{U_{E}^{2}}\right),
$$




$$
c_{p 04}^{T}=-\frac{U_{E}^{2}}{U_{m v}^{2}}\left[\left(1-c_{p e 4}\right)+4\left(1+k_{d}\right) \frac{\dot{U}_{E} R}{U_{E}^{2}}\right],
$$

where $k_{d}$ is the dimensionless position of the detachment point of the fluid flow, placed at $x=x_{d}=R k_{d}\left(k_{d}<1\right)$ in steady flow case, $U_{E}$ is the unsteady fluid flow velocity as a time function in $\mathrm{m} / \mathrm{s}$, and $\dot{U}_{E}$ represents the derivative with reference to time $\left(\dot{U}_{E}=\dot{U}_{E}^{F}\right)$ eqn. (4).

\subsection{Empirical estimation of the $k_{m}$ and $k_{d}$}

The empirical parameters $k_{m}$ and $k_{d}$, can be used to fit the theoretical stagnationpressure referenced coefficients, $c_{p 01}^{T}$ and $c^{T}{ }_{p 04}$ to the experimental results, $c_{p 01}^{E}$ and $c_{p 04}^{E}$. For estimating the $k_{m}$ and $k_{d}$ values, the standard deviation of the experimental results are used. The definition of the standard deviation, $\sigma$, is

$$
\sigma_{x}=\sqrt{\frac{1}{N-1} \sum_{i=1}^{N}\left(x_{i}-\bar{x}\right)^{2}},
$$

where $N$ is the number of terms in the data vector, $\bar{x}$ the mean value of the vector and $x_{i}$ the value of the vector at the position of $i=0,1,2,3$.. and $x$ represents the $c_{p 01}$ or $c_{p 04}$.

By using a Matalb program, with an initial value of the, $k_{m}, k_{d}=0$ and $c_{p e 4}=$ -0.5 , the values of the $c_{p 01}^{T}, c_{p 04}^{T}, \sigma_{01}^{T}$ and $\sigma_{04}^{T}$ are calculated by using eqn. (18). These results are compared with the experimental results $c_{p 01}^{E}, c_{p 04}^{E}, \sigma_{01}^{E}$ and $\sigma_{04}^{E}$. The values of $k_{m}$ and $k_{d}$ are in counted until the difference between, $\sigma_{01}^{T}$ and $\sigma_{01}^{E}$, and $\sigma_{04}^{T}$ and $\sigma_{04}^{E}$ are $\leq 0.001$. The values of $k_{m}$ and $k_{d}$ obtained are shown in figs. 9 and 10 .

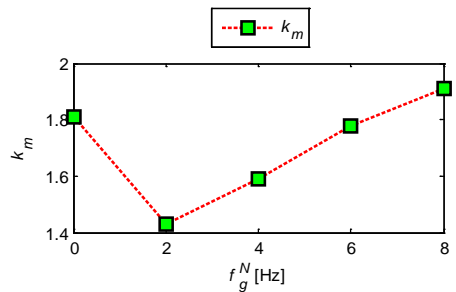

Figure 9: Variation of maximum speed parameter, $k_{m}$, with the nominal gust frequency $f_{g}^{N}$.

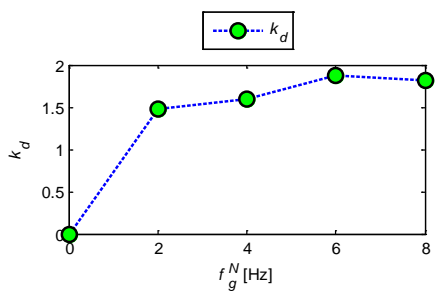

Figure 10: Variation of detachment point parameter, $k_{d}$, with the nominal gust frequency $f_{g}^{N}$. 


\subsection{The influence of the unsteady flow}

The stagnation-pressure referenced coefficients obtained from the theoretical model are the summation of a steady and an unsteady flow terms eqns. (16) and (17). The steady flow terms $\left(c^{T}{ }_{p 01 \mathrm{~S}}\right.$ and,$\left.c^{T}{ }_{p 04 \mathrm{~S}}\right)$ and unsteady flow terms $\left(c^{T}{ }_{p 01 \mathrm{U}}\right.$ and $c_{p 04 \mathrm{U}}^{T}$ ) defined as follows

$$
\begin{array}{ll}
c_{\mathrm{p} 01 \mathrm{~S}}^{T}=-\frac{U_{E}^{2} k_{m}^{2}}{U_{m v}^{2}}, & c_{\mathrm{p} 04 \mathrm{~S}}^{T}=-\frac{U_{E}^{2}\left(1-c_{p e 4}\right)}{U_{m v}^{2}}, \\
c_{\mathrm{p} 01 \mathrm{U}}^{T}=\frac{4 \dot{U}_{E} R}{U_{m v}^{2}}, & c_{\mathrm{p} 04 \mathrm{U}}^{T}=\frac{4\left(1+k_{d}\right) \dot{U}_{E} R}{U_{m v}^{2}} .
\end{array}
$$

In this way, it is possible to show the contribution of the unsteady terms.

Results of steady and unsteady flow theoretical terms, and experimental measurements concerning stagnation pressure referenced coefficients are plotted in fig. 11 and fig. 12. Results are discussed in section 7.

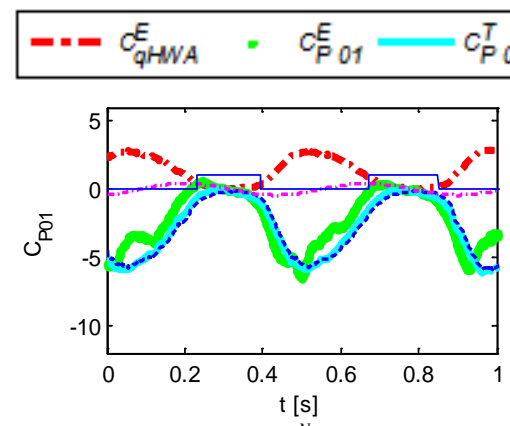

(a): $f_{g}^{N}=2 \mathrm{~Hz}$

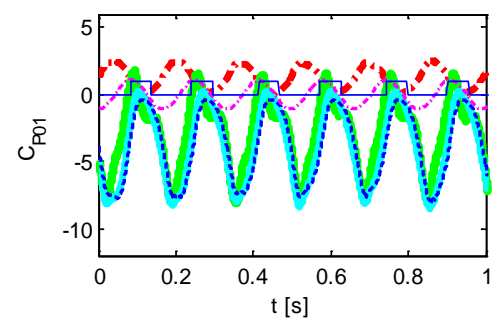

(c): $f_{g}^{N}=6 \mathrm{~Hz}$

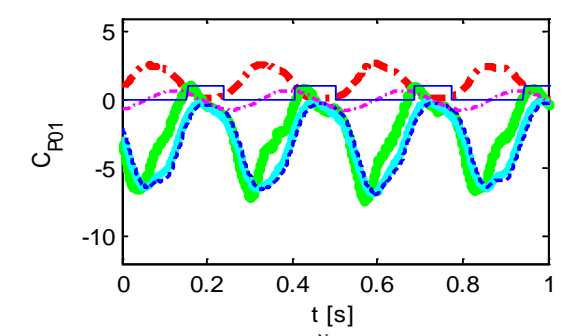

(b): $f_{g}^{N}=4 \mathrm{~Hz}$

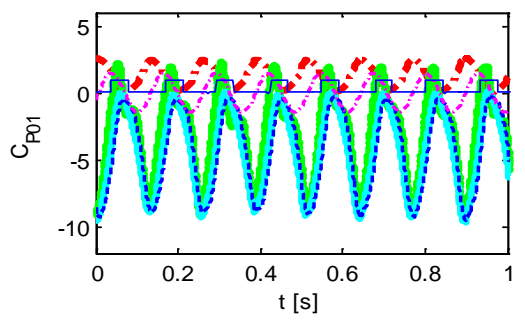

(d): $f_{g}^{N}=8 \mathrm{~Hz}$

Figure 11: Plot of the $c^{E}{ }_{q H W A}, c^{E}{ }_{p 01} c_{p 01}^{T}, c^{T}{ }_{p 01 S}$ and $c^{T}{ }_{p 01 \mathrm{U}}$ and gust generating gate position (1: is fully open, 0 : closed) varies with time function $\mathrm{t}[\mathrm{s}]$, in configuration $\mathrm{C}_{4}$, with rotating gate chord, $T_{g c}=380 \mathrm{~mm}$, nominal gust frequency, $f_{g}^{N}=[2,4,6,8 \mathrm{~Hz}]$ and fan speed, $f_{m}=40$ $\mathrm{Hz}$ (i.e. mean flow speed is 6 to $7 \mathrm{~m} / \mathrm{s}$ ). 


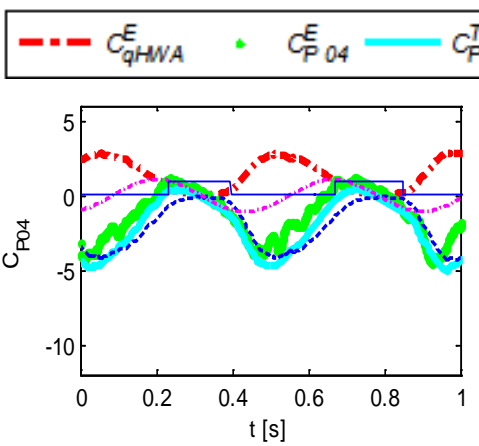

(a): $f_{g}^{N}=2 \mathrm{~Hz}$

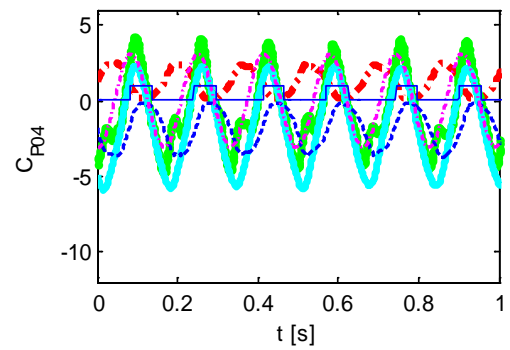

(c): $f_{g}^{N}=6 \mathrm{~Hz}$

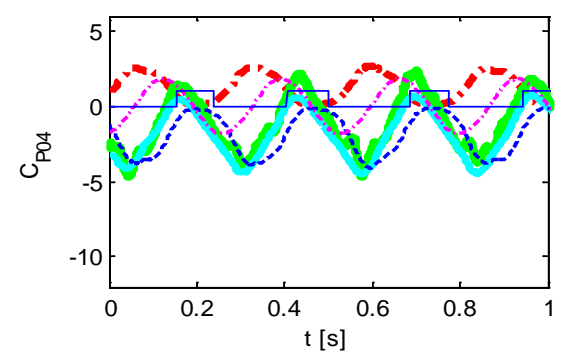

(b): $f_{g}^{N}=4 \mathrm{~Hz}$

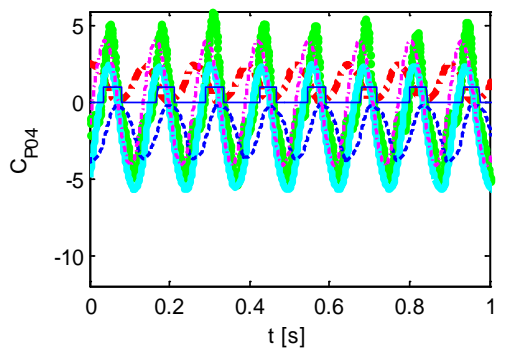

(d): $f_{g}^{N}=8 \mathrm{~Hz}$

Figure 12: Plot of the $c^{E}{ }_{q H W A}, c_{p 04}^{E}, c_{p 04}^{T}, c_{p 04 S}^{T}$ and $c_{p 04 \mathrm{U}}^{T}$ and gust generating gate position (1: is fully open, 0 : closed) varies with time function $\mathrm{t}[\mathrm{s}]$, in configuration $\mathrm{C}_{4}$, with rotating gate chord, $T_{g c}=380 \mathrm{~mm}$, nominal gust frequency, $f_{g}{ }^{N}=[2,4,6,8 \mathrm{~Hz}]$ and fan speed, $f_{m}=$ $40 \mathrm{~Hz}$ (i.e. mean flow speed is 6 to $7 \mathrm{~m} / \mathrm{s}$ ).

\section{Discussions on results}

- A gust wind tunnel has been built at (IDR/UPM), successfully equipped with the new hardware (a gust generation frequency controller, hot-wire anemometer probe).

- Pitot tube and hot-wire anemometer probe measurements are in close agreement in the steady flow (fig. 4).

- The characterization of the gust wind as a sinusoidal variation has been satisfactory (the regression coefficient, $\mathrm{R}^{2}$ is close to 1 ). The gust frequency inside the test section, $f_{g}{ }^{F}$, is similar to the nominal gust frequency, $f_{g}^{N}$, (shown in fig. 5 and table 2).

- In the case of uniform unsteady flow, the proposed correction term, $T_{c f}$, gives a good approximation of the Pitot tube measured experimental values up to a nominal gust frequency is, $f_{g}^{N}=4 \mathrm{~Hz}$ (figs. 6 (a) and 6(b)). If the nominal gust frequency is, $f_{g}{ }^{N}>4 \mathrm{~Hz}$, the term $T_{c t}$ gives no good correction (figs. 6(a) and 6(b)), which could be due to other phenomena like internal configuration of the Pitot tube (which needs further study). 
- In the case of uniform unsteady flow, the study of the influence of the static pressure position along with the wind tunnel tesction- 2 base wall shows that, for all nominal gust frequencies $f_{g}{ }^{N} \leq 8 \mathrm{~Hz}$, with the experimental setup (fig. 7), the phase of the correction is in the right side. if $x_{e \mathrm{PT}}$ value $>0$, the correction amount is a bit excessive (fig. 8(d)), if $x_{e \mathrm{PT}}$ value $=0$ then no correction is required (fig. 8(c)) and if $x_{e \mathrm{PT}}$ value $<0$ the correction amount is enough to simulate the Pitot experimental measurements.

- In the case of the flow around a semi-circular cylinder placed in the wind tunnel test section, in steady flow $\left(f_{g}=0 \mathrm{~Hz}\right)$, the experiments (NavarroMedina [2]) proposed that the values of $k_{m}=1.73$ and $k_{d}=0$. But as gust frequency increases these values fails to fit the satisfactory experimental and the theoretical results. The empirical method is developed an estimation of the $k_{m}$ and $k_{d}$ values, which allows a better agreement between experimental and theoretical predications.

- As shown in figs. 9 and 10 , the empirical parameters $k_{m}$ and $k_{d}$ values changes as a function of nominal gust frequency, $f_{g}{ }^{N}$.

- For all the nominal gust frequency range tested $0<f_{g}{ }^{N}<8 \mathrm{~Hz}$, the theoretical predictions are in good agreement with the experimental results. The unsteady terms correctly contributes to the good fitting (figs. 11 and 12). The amount of this term is not negligible for nominal gust frequency $f_{g}^{N} \geq 4 \mathrm{HZ}$.

\section{Conclusions}

In this paper, some results concerning the velocity and pressure measurements in unsteady flow performed in a gust wind tunnel, have been presented. The gust flow in the wind tunnel employed (developed at IDR/UPM) has been characterized, showing a nearly sinusoidal velocity variation with time. Concerning the unsteady effects the relevance correction term for unsteady flow measuring instrument (Pitot tube) has been obtained. A theoretical model has been developed based on potential flow theory, which helps to explain the contributions of the unsteady flow in the case of a semi-circular cylinder.

The theoretical model presented efficiently allows to analysing the steady and unsteady flow terms with various frequencies of the uniform gusty winds.

\section{References}

[1] Sanz-Andres, A. and Navarro-Medina, F., The initiation of rotational motion of a lying objects caused by wind gusts. J. Wind Eng. Ind. Aerodynamics, 98, pp. 772-783, 2010.

[2] Navarro-Medina, F., Sanz-Andres, A. and Perez-Grande, I., Gust wind tunnel study on ballast pick-up by high-speed trains. Experiments in fluids, 121, pp. 52-105, 2012.

[3] Pratt, M., Principles of Flight, Airplane Flight Equipment Limited: Manchester, pp. 108-111, 2004. 
[4] Naudascher, I., and Rockwell E.D., Flow Induced Vibrations an Engineering Guide, Dover Publications: Mineola, New York, pp. 238-244, 1994.

[5] Dantec Dynamics, http//www.dantecdynamics.com

[6] Meseguer, J. and Sanz-Andres, A., Aerodinámica basica $2^{a}$ Edicion, Ibergarceta publications, S.L: Madrid, pp. 35-50, 2011.

[7] Batchelor, G.K., An introduction to fluid dynamics, Cambridge University Press, pp. 121-131, 1973.

[8] Roshko, A., On the drag and shedding frequency of two-dimensional bluff bodies, NACA-TN-3169, 1954.

[9] Clancy, L.J., Aerodynamics (Chapter 3). Topics on pressure fields, Pitman Publishing Limited: London, pp. 22-59, 1975. 